\title{
Where to Place Cameras on a Snake Robot: Focus on Camera Trajectory and Motion Blur
}

\author{
Mehmet Mutlu ${ }^{1}$, Kamilo Melo ${ }^{1}$, Massimo Vespignani ${ }^{1}$, Alexandre Bernardino ${ }^{2}$ and Auke Jan Ijspeert ${ }^{1}$
}

\begin{abstract}
Visual information is heavily used in robotics, in particular for SLAM applications. Visual SLAM algorithms depend on robust feature extraction and reliable state estimation. Quality of the visual information highly depends on how that information is captured. The nature of snake robots' locomotion presents considerable challenges on the quality of images captured by an onboard mobile camera. Although placing the camera on the "head" of the snake robot has advantages when the robot is stationary since the body can be used as a manipulator observing for the environment, how to place the camera in order to capture more useful images for navigation during locomotion is not clear. In this paper, we present a comparative study to discuss implications of the camera location on field coverage and types of image quality for three snake gaits: Rolling, sidewinding and linear progression. Camera pose during locomotion is examined in detail and quality of images are quantified using a motion blur metric which relates camera egomotion to blur. Linear progression is found to be very promising in terms of supplying sharper images. But, there are also other merits that can be exploited in different locomotion types and camera locations.
\end{abstract}

\section{INTRODUCTION}

In the literature related to robotic search and rescue (SAR), it is common to find mentions to "Snake robots" as potential tools for accessing narrow cavities in places that any other platform cannot reach [1]. With the exception of the Active Scope Mechanism [2], to the date, there is a lack of deployments of such technology in real missions due to either (i) the level of readiness of the snake robot platforms [3]-[5], their cost and complexity [6], [7], or simply because they do not comply with regulations needed to operate in certain environments [1]. We consider that there is still a more relevant factor that delays the introduction of these robots as real assets for SAR missions. The definition of a task that a snake robot can perform better than other robots is still fuzzy. For instance, tasks like assessing the structural status of a collapsed building with limited access, provide video feedback of interest points (including possible victims) where a borehole is the only access through the rubble, and so on, omit many details of how the robot will perform the task with limited perception equipment on board or how capable the remote operator is (or the robot itself if it is meant to be autonomous) to control the motion and task execution.

\footnotetext{
${ }^{1}$ M. Mutlu, K. Melo, M. Vespignani and A. J. Ijspeert are with Biorobotics Laboratory, School of Engineering and Institute of Bioengineering, Ecole Polytechnique Fédérale de Lausanne, $\mathrm{CH}-$ 1015 Lausanne, Switzerland mehmet.mutlueieee.org, kamilo.melodepfl.ch

${ }^{2}$ A. Bernardino is with Electrical and Computer Engineering of the Faculty of Engineering at IST, Instituto Superior Téchnico, 1049-001 Lisbon, Portugal alexandre.bernardino@tecnico.ulisboa.pt
}

As perception become crucial in these types of tasks, as many times mentioned in the literature, the use of a snake robot as a mobile extension of a camera [2] prevails. In fact, several researchers had come with this idea and intuitively incorporate a camera in the "head" of their snake robot designs [4]-[7]. Two possible ways to exploit a camera mounted on the head of the robot are for video surveillance, and for navigation. In the former, the camera acts as a simple final tool of a redundant manipulator, operated once the robot is positioned in place while guaranteeing some level of mechanical stability. The later, is more complex, as it proposes an interplay between computer vision algorithms and locomotion controllers for snake robots. To the best of our knowledge, there is scarce literature available for the mentioned topics. The references above report some robot implementations that incorporate a camera in the head module. However, there is no information on their effective use to provide data for navigation during locomotion.

Nevertheless, related work is available in the control of a snake robot, to stabilize the motion of the robot's end modules as a gait progresses [8], [9]. Particularly, in [9], optimization techniques are used to the problem of moving a snake robot (an expensive system) optimizing simultaneously the gait performance as well as the ability to maintain motion of a specific module in certain way i.e. keeping the head module with minimal changes in their orientation as the gait progresses. These algorithms effectively control the robot with the expected results obeying defined policies, however their use with a camera to acquire useful video/image data for self-navigation is missing. On the other hand, in [10], the authors use the Modular Snake Robot Lola-OP ${ }^{\mathrm{TM}}{ }^{1}$, with a camera added in the head module, running standard video stabilization algorithms in order to correct the video as the robot moves. But there is no mention of neither coordination of video processing during the actual robot gaits, nor any video database useful for navigation.

All these aforementioned implementations of camera-onhead of a snake robot rely on the fact that the robot is a serial manipulator featuring a slender form and small cross section. Thus, all these robots incorporate their cameras intuitively placed pointing along the longitudinal axis of the robot. The question we want to address in this paper is: is this camera placement in a snake robot (i.e. on the head and pointing longitudinally) in fact optimal for navigation as it seems to be for inspection and how does this depend on the type of the gait?

${ }^{1}$ Developed by KM-RoBoTa s.a.s. (http://km-robota.com) 
We want to provide a systematic approach for studying the placement of a camera in a Modular Snake Robot (MSR) since the use of a snake robot as a camera manipulator in order to acquire video data of a selected scene is straightforward. The idea behind our study is twofold. In the first place, we are simultaneously observing the natural coverage of camera with respect to absolute motion of the MSR for different gaits and for different camera locations. The second idea is tracking motion blur amount in images captured by an onboard camera during the same gaits and for the same proposed camera locations. On the other hand, our interest to find a correlation between camera trajectories and the video motion blur is because of the fact that a MSR like Lola$\mathrm{OP}^{\mathrm{TM}}$, shown in figure 1, is capable of "holonomic" and repeatable motions. Additionally, the payload (weight and volume) of a MSR is not always constrained. This makes worth the analysis of different locations as well as different orientations of a fixed camera in a MSR, in order to explain quantitatively where and under which conditions a camera should be mounted, for maximizing the information gathered and ensuring quality for the processing.

The outline of this paper is as follows. In Sec. II, the description of the robot, the gaits used, the camera position and orientation are shown. Then, in Sec. III the expected motion blur of images captured by the camera while MSR is performing locomotion is reported. Further implications of motion blur and locomotion correlation is discussed in Sec. IV and some real life deployment examples are given. Finally, conclusions are given in the last section.

\section{CAMERA TRAJECTORY DURING LOCOMOTION}

A common framework to control modular snake robot's locomotion is provided by [11].

$$
Q(n, t)= \begin{cases}O_{e}+A_{e} \sin \left(w_{e}+n / \lambda_{e}+\delta\right) & \text { if } n \text { is even } \\ O_{o}+A_{o} \sin \left(w_{o}+n / \lambda_{o}\right) & \text { if } n \text { is odd }\end{cases}
$$

In Eq. (1), $O$ is the offset angle that represents the center of oscillation, $A$ is the amplitude of oscillation, $w$ is the oscillation frequency, $\lambda$ is a body wave number and $\delta$ is the phase difference between horizontal and vertical joints.

Depending on the application, the operator may need to keep the line of sight fixed to a certain area or try to observe large space during locomotion. Observing the trajectory of the camera for different types of gaits gives an idea about the coverage of the camera. There exists a trade-off in the stability of the image and covered area. Depending on the requirements of the application the definition of optimal camera placement will differ. Hence, a comparative study will be presented for camera field of view and pose.

Different gaits of the MSR are simulated in Webots 7.4.3. In Fig. 1, coordinate frames of the simulation world and local sensors that are placed on each module can be seen.

Rolling, sidewinding and linear progression are three main gaits of snake robots. In this paper, one example from each of rolling, sidewinding and linear progression will be studied.

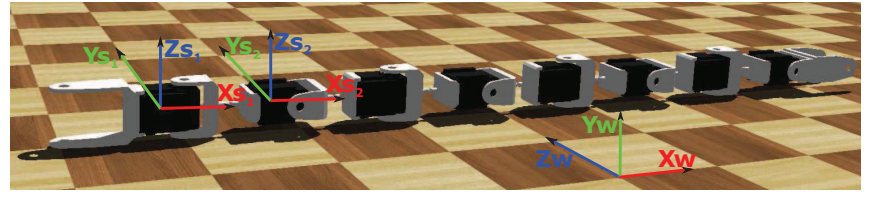

Fig. 1. Modular Snake Robot Lola-OP ${ }^{\mathrm{TM}}$ showing coordinate frames of global world and local sensors in a simulation environment.

TABLE I

PARAMETERS AND COORDINATE TRANSFORMATIONS OF CAMERA PLACEMENT FOR EXAMINED GAITS

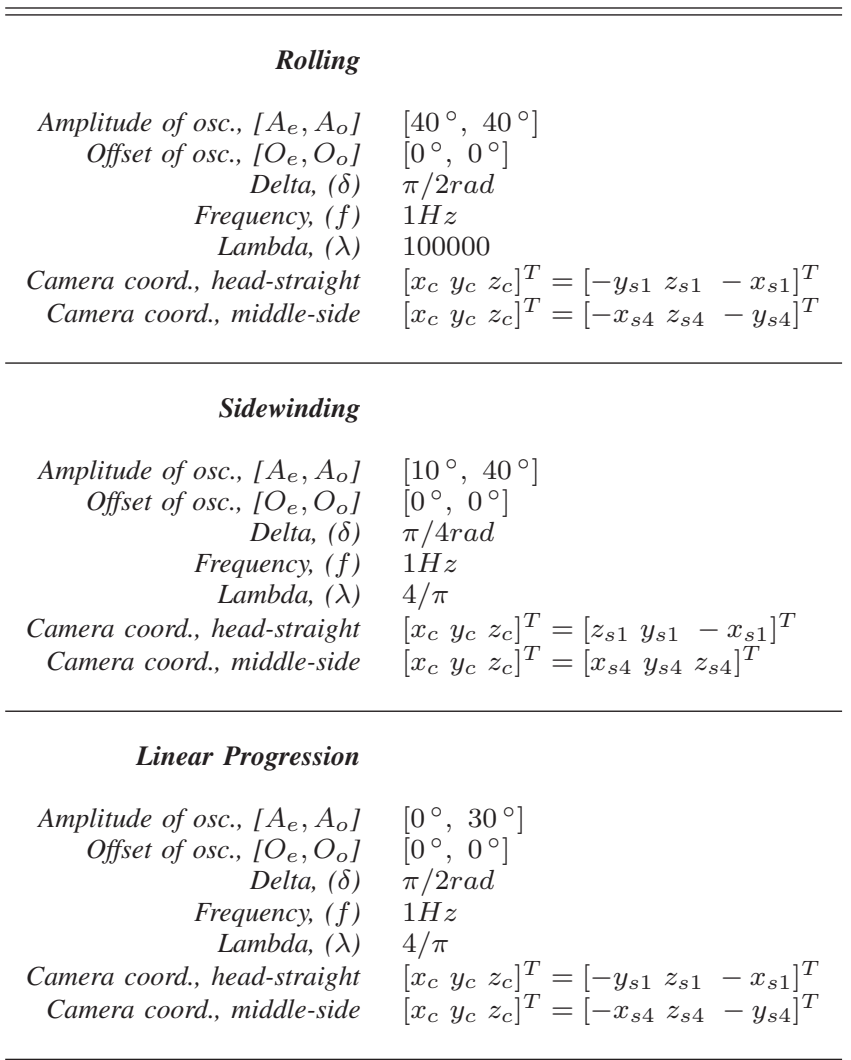

Frequency of the gaits, $f$, is chosen to be same for even and odd modules and for all gaits and it is set to $1 \mathrm{~Hz}$. All gait parameters for all experiments can be seen in the Table I. For each of the locomotion examples, the camera is assumed to be placed in two separate locations. Either, looking straight head camera or middle camera pointing towards sideways with respect to the snake longitudinal axis. The head camera is placed on the same location as the first sensor coordinate frame shown on the Fig. 1 with the orientations given in Table I. Similarly, the middle camera is placed on the fourth module with the orientations given in Table I.

For visualizing the camera field of view, pose of the each module of the snake robot is logged for short duration. In figures from Fig. 2 to Fig. 7 trajectories of all 8 modules of the snake are shown. The color of a single module's path is drawn as changing from black to specific color. The very first black dot in the beginning of each module's path is captured at $t=0 \mathrm{sec}$ and position of the module is logged for three or 
two (only linear progression) seconds for each run. The color change from black to a saturated color progresses linearly from $t=0$ to end of the run. Each module's path is plotted with a different color starting with red on the head, followed by green on the second module, blue for the third one and so on. The pose of individual modules are logged in $1 \mathrm{kHz}$. However, position of the modules are illustrated with dots which are drawn 50ms apart on the plots. Hence the density of the dots throughout a path gives an intuition about the linear velocity of the module. The denser the dots are, the longer the module stays around those dots which means that module moves relatively slow on that region. The overall snake velocity is marked with a blue arrow in each figure. In addition to the trajectory of modules, the orientation of the camera is also shown throughout the locomotion in figures from Fig. 2 to Fig. 7. Orange lines starting from the camera node represent the primary axis of the camera $(\mathrm{z})$ and are oriented along the camera's viewing direction. The actual field of view depends on lenses and is application-specific, whereas the camera direction is universal for all applications. The color gradient of camera's primary axis lines, from black to orange, has the same time progress notion with path of the snake's module position. Camera pose is plotted with fine sampling through the end and coarsely in the beginning of each run. Coarse parts also have coordinate axes plotted on them. Each plot also has zoomed versions of camera pose for the first second of locomotion.

\section{A. Rolling motion}

The rolling motion of a snake robot involves continuous rotation of the body around longitudinal axis, as can be seen in Fig. 2 and Fig. 3 for three seconds. In these figures, the robot is moving on $\mathrm{x}-\mathrm{z}$ plane where $\mathrm{y}$ axis is the height from ground. The robot takes an arch shape to move sideways when offset terms of Eq. (1) are zero. This motion is known to be robust in rough terrain and slopes [12].

1) Head camera looking straight: The first observation that can be made about placement of the camera on the head looking away in the longitudinal direction of the snake is that the camera is not pointing towards the motion direction. Center of the field of view of a camera is looking more than 90 degrees away from it as it can be seen in Fig. 2 . This means the robot would not know about most of the approaching obstacles. Moreover, it is not easy to guide the robot with the information obtained from captured images if the camera is pointing away from the locomotion direction.

Camera field of view stays quite close to previous field of views. However, continuous rolling of the snake means rolling of the camera around itself which can be seen clearly on the zoomed part of Fig. 2. Hence the view would be rolling and not stay very stable while rolling.

2) Middle camera looking sideways: Placing the camera on the fourth module of the robot with orientation to point sideways results in very large displacement of the field of view between image frames during rolling. Although camera motion that is shown in Fig. 3 would be naturally covering a large area like a torus around the snake, camera rotation

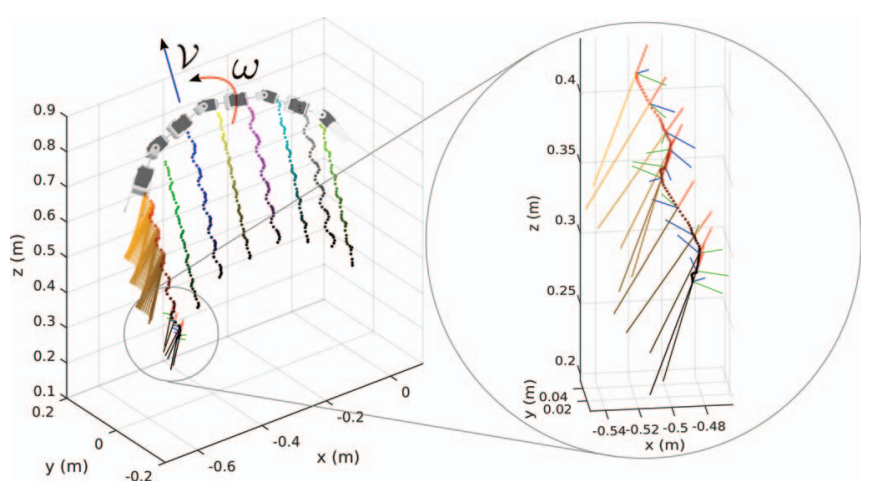

Fig. 2. Camera and snake trajectory during rolling gait. Camera is placed on head looking straight.

velocity is quite fast. Large rotational velocity results in large displacements of pixels on the image plane during the exposure period which usually has negative effect on visual SLAM algorithms and can disturb convergence of SLAM.

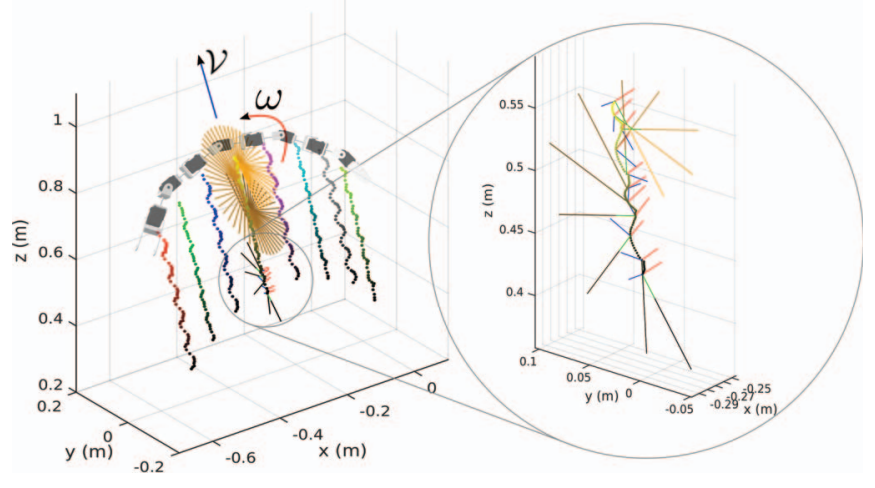

Fig. 3. Camera and snake trajectory during rolling gait. Camera is placed on the fourth module looking sideways.

\section{B. Side-winding motion}

Sidewinding is a very fast gait. Similar to rolling, motion of the robot is directed sideways, with a small forward component. However, the number of contact points with the ground is much smaller than rolling motion. Sidewinding is commonly performed by snakes moving on granular medium and surface contact points of the snake follows discontinuous trajectories. Slight oscillatory motion, as seen on Fig. 4 and Fig. 5 is mainly caused by the asymmetrical structure of the snake robot used in the simulations. Sidewinding plots span three seconds of experimental data.

1) Head camera looking straight: The camera placement in this case is similar to the rolling case. Fig. 4 shows camera points mostly perpendicular to the direction of locomotion. However, camera orientation does not have continuous rotation component unlike the rolling gait. Also the average speed of the sidewinding is higher than rolling. Translational progress of the camera is not uniform in sidewinding. Camera stays around ground contact point of the head module for a while and moves quite fast within the swing phase. 


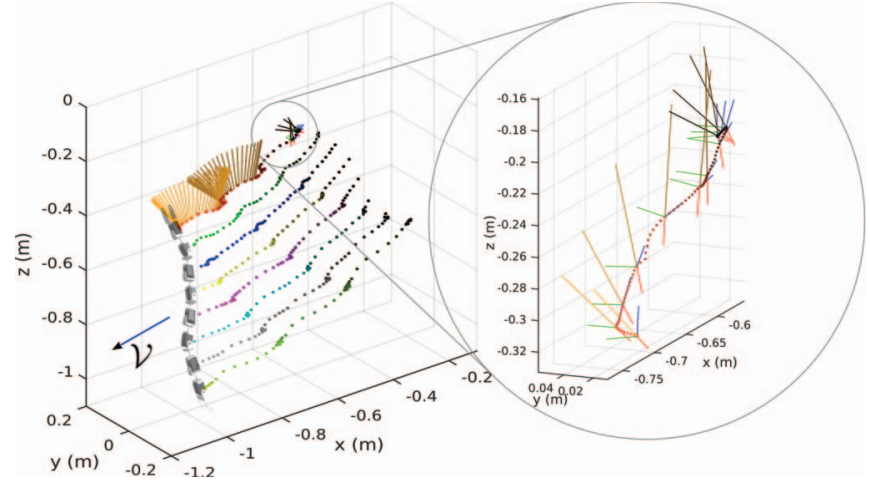

Fig. 4. Camera and snake trajectory during sidewinding gait. Camera is placed on head looking straight.

2) Middle camera looking sideways: When the camera is mounted on the direction of the locomotion in a middle module, it can be quite informative for sidewinding gait. It is not only pointing through the locomotion direction, but also exhibiting a circular exploratory motion around the snake direction, even though exploration may not cover a wide area. Furthermore, orientation of the camera always stays around the initial orientation. This type of camera placement can be useful to teleoperate the robot during locomotion in a remote place.

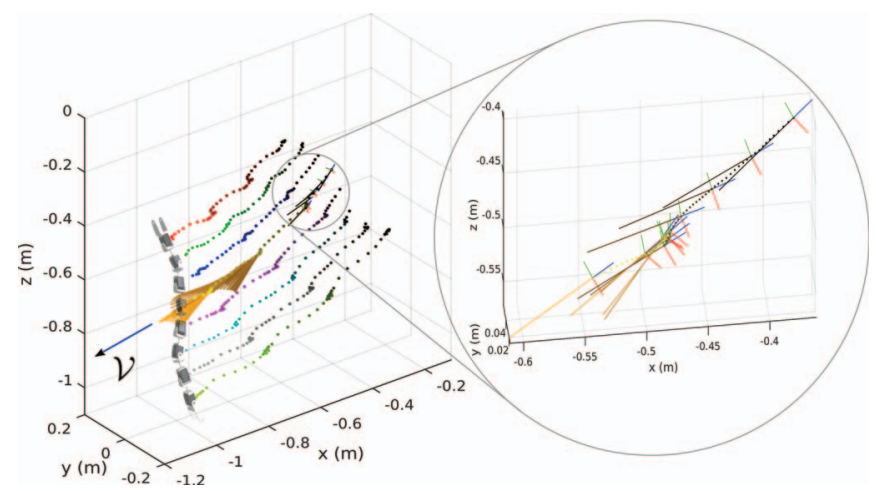

Fig. 5. Camera and snake trajectory during sidewinding gait. Camera is placed on the forth module looking sideways.

\section{Linear progression motion}

Linear progression is examined as the last type of gait in this study. Linear progression can be obtained with horizontal waves and vertical waves. However, due to high friction between ground and snake, horizontal waves cause either too much energy loss or do not result in proper locomotion. Therefore, only vertical wave generated linear progression is considered in this part. The average speed of the robot is much slower than rolling and sidewinding while performing linear progression. Fig. 6 and Fig. 7 covers only two seconds of data, because position samples of consecutive modules start to overlap for longer runs.

1) Head camera looking straight: The most noticeable characteristic of camera motion in this gait and placement is that there is almost no rotation with respect to two of the axes. Dominant rotation is mainly on pitch. Also the translational speed of the camera is quite low which makes the camera quite steady. The field of view is oscillatory around an offset and on the direction of motion, which helps to control the robot in teleoperated situations.

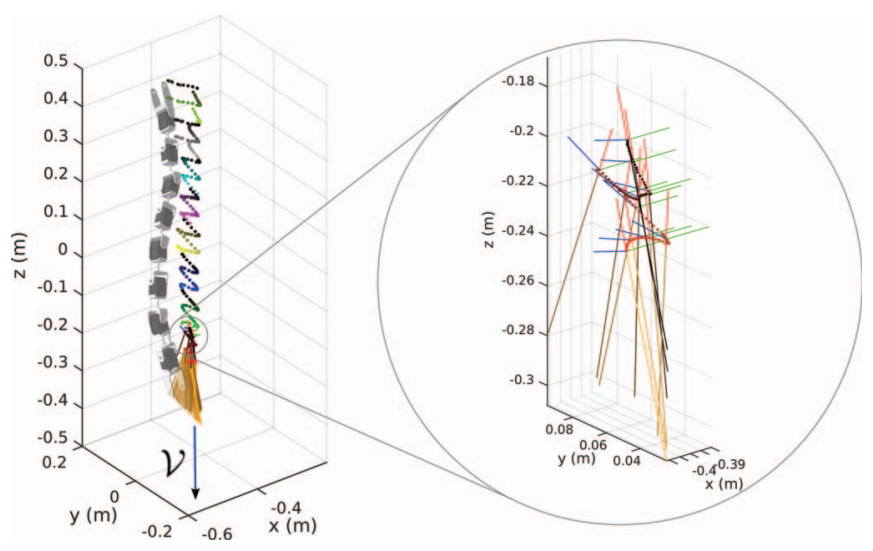

Fig. 6. Camera and snake trajectory during linear progression gait. Camera is placed on head looking straight.

2) Middle camera looking sideways: When the camera is mounted on the middle part of the snake and pointing towards sideways, camera motion is minimal and there is only slow oscillatory roll and translational motion of the camera. Also the region that the camera observes is quite stable.

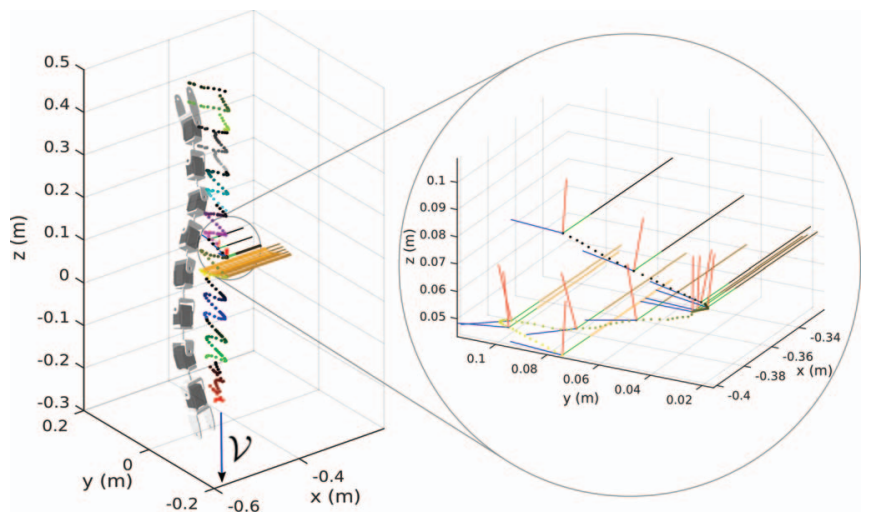

Fig. 7. Camera and snake trajectory during linear progression gait. Camera is placed on the fourth module looking sideways.

\section{MOTION BLUR OF CAMERA DURING LOCOMOTION}

In vision applications, the quality of captured images is equally important as the trajectory of the camera. Most of the SLAM applications require sharp images to extract features correctly, the abundance of features and low pixel displacement during locomotion. In many cases, features are extracted from the texture of surrounding environment. That stage may require engineering the environment, if the natural texture is poor. In realistic search and rescue missions, it is too hard and time consuming to modify the environment. Also, there can be insufficient light in the environment 
that would push the camera to capture images at higher exposure time, resulting in higher motion blur. However, capturing relatively sharper images by examining motion of the robot is possible. Motion blur is one of the most pronounced causes of distortions in robot vision. Certain amount of motion blur can be recovered with proposed motion deblurring algorithms in the literature [13]. However, when the amount of motion blur is extreme, information loss can be irreversible. One of the most common methods to avoid motion blur is using stabilization platforms [14]. The structure and size of snake robots are usually not well suited for adding mobile stabilization platforms. On snake robots, even using the first few degrees of freedom for camera stabilization instead of locomotion can be effective up to a certain degree. However, one of the biggest challenges in snake locomotion is the state estimation due to complex ground-robot interaction. Furthermore, high vibrations and impacts observed on the body makes visual SLAM on snake robots during locomotion a very hard problem [15].

In this part of the paper, motion blur formation during snake locomotion will be analysed and motion blur characteristics of different gaits will be explained. Motion blur can be caused by the movement of objects on the scene or movement of the camera. Although there can be exceptions, the environment is expected to be mostly stationary for the robotics applications in a search and rescue area. This study addresses motion blur only caused by the egomotion of the camera. The effect of rotation and translation motions are different on motion blur. In particular, the amount of motion blur caused by translation depends on scene depth in addition to translational velocity, but motion blur caused by rotational motion has no scene depth dependence. Furthermore, measuring translational speed on a snake robot is more challenging than measuring rotational speed. Despite, a simple MEMS gyroscope can directly give rotational speed, translational speed can be estimated from the integration of accelerometer measurements in which calibration and bias errors accumulate. Also, the effect of translation on motion blur can be negligible when the scene depth is sufficiently large. In open fields, scene depth is large and makes the translational motion blur relatively small. Even though snake robots may need to work in cluttered environments where scene depth is small, we will only consider motion blur caused by the rotational motion of the snake. One specific observation is that rotational over translational speed ratio of the body elements on a snake robot is higher than most of the conventional wheeled or tracked robots due to natural movement of snakes. Therefore, considering only the effect of rotational motion on motion blur will still address significant amount of the motion blur source in snake robots.

A motion blur metric, Motion-Based Motion Blur Metric (MMBM), which estimates the amount of motion blur on a camera undergoing rotational motion is given in [16]. Higher values of the metric corresponds to higher blur. The proposed metric models optical flow of pixels out of rotational speed measurements and approximates the motion blur that would be observed on images. The definition of $\operatorname{MMBM}(\mu)$ is given as

$$
\mu:=\frac{1}{\Delta u \Delta v} \int_{u_{\min }, v_{\min }}^{u_{\max }, v_{\max }} \sqrt{\dot{u}^{2}+\dot{v}^{2}} \mathrm{~d} v \mathrm{~d} u,
$$

where $\mathrm{u} \& \mathrm{v}$ are image sensor coordinates, $\Delta u \& \Delta v$ are image sensor size and $\dot{u} \& \dot{v}$ are optical flow vectors derived from gyroscope measurements in following way,

$$
\begin{aligned}
\dot{u}^{2}+\dot{v}^{2}=\frac{w_{y}^{2}}{f^{2}} \boldsymbol{u}^{\mathbf{4}} & +\frac{w_{x}^{2}}{f^{2}} \boldsymbol{v}^{\mathbf{4}}-2 \frac{w_{x} w_{y}}{f^{2}} \boldsymbol{u}^{\mathbf{3}} \boldsymbol{v}-2 \frac{w_{x} w_{y}}{f^{2}} \boldsymbol{u} \boldsymbol{v}^{\mathbf{3}} \\
& +\frac{w_{x}^{2}+w_{y}^{2}}{f^{2}} \boldsymbol{u}^{\mathbf{2}} \boldsymbol{v}^{\mathbf{2}}+\left(2 w_{y}^{2}+w_{z}^{2}\right) \boldsymbol{u}^{\mathbf{2}} \\
& +\left(2 w_{x}^{2}+w_{z}^{2}\right) \boldsymbol{v}^{\mathbf{2}}-4 w_{x} w_{y} \boldsymbol{u} \boldsymbol{v}-2 w_{x} w_{z} f \boldsymbol{u} \\
& -2 w_{y} w_{z} f \boldsymbol{v}+\left(w_{x}^{2}+w_{y}^{2}\right) f^{2},
\end{aligned}
$$

where $f$ is the focal length of the camera and $\left[w_{x}, w_{y}, w_{z}\right]$ is rotational velocity of the camera. Authors also show a $\mu$ based real-time image capturing technique to reduce the average amount of motion blur on captured images from a camera mounted on a six-legged robot RHex in [16]. In this current work, $\mu$ will be used for motion blur characterization. The value of $\mu$ assumes frames captured with unit exposure time. If the value of exposure is not fixed in the application $\mu$ needs to be scaled with the exposure time. We also consider fixed exposure time. $\mu$ will be calculated for the different snake gaits examined in Sec. II.

\section{A. Rolling motion}

Unlike most of the legged straight locomotion cases, snake rolling involves continuous rotation as seen in $w_{z}$ and $w_{x}$ parameters of Fig. 8 and Fig. 9 respectively.

1) Head camera looking straight: When the camera is on the head and looking straight during rolling, there is quite dominant rolling $\left(w_{z}\right)$ motion of camera. Although there are also oscillations on yaw $\left(w_{z}\right)$ and pitch axis $w_{x}$, the resulting motion blur has small oscillations around certain value. In roll dominated camera images, center of the images would be sharper and the periphery would be more blurred. Having some sharper region around the middle parts can be exploited in vision tasks.

2) Middle camera looking sideways: When Fig. 8 and Fig. 9 are compared, the similarity in the motion of head module and middle module can be easily seen. Main difference appears in the axes assignment to camera motion due to camera direction change. When high continuous rotation axis of the snake robot is assigned to the pitch axis of the camera, the amount of motion blur significantly increases. This happens because pitch motion creates stronger motion blur on whole image compared to roll of camera. Hence, this particular camera placement results in excessive motion blur during snake rolling gait.

\section{B. Side-winding motion}

Sidewinding exhibits high speed swing phases and relatively stationary stance phases for each module. Unlike the 


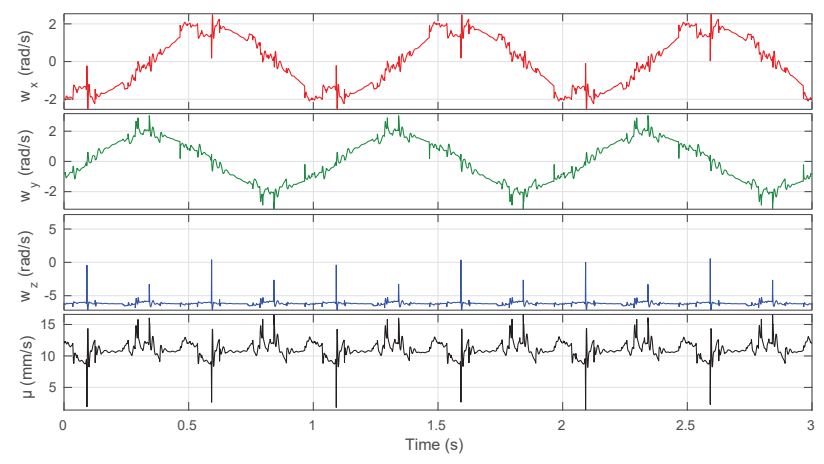

Fig. 8. $\operatorname{Roll}\left(w_{z}\right), \operatorname{pitch}\left(w_{x}\right)$ and $\operatorname{yaw}\left(w_{y}\right)$ rotational speeds of camera and corresponding $\mu$ when straight pointing camera is placed on head of a rolling snake.

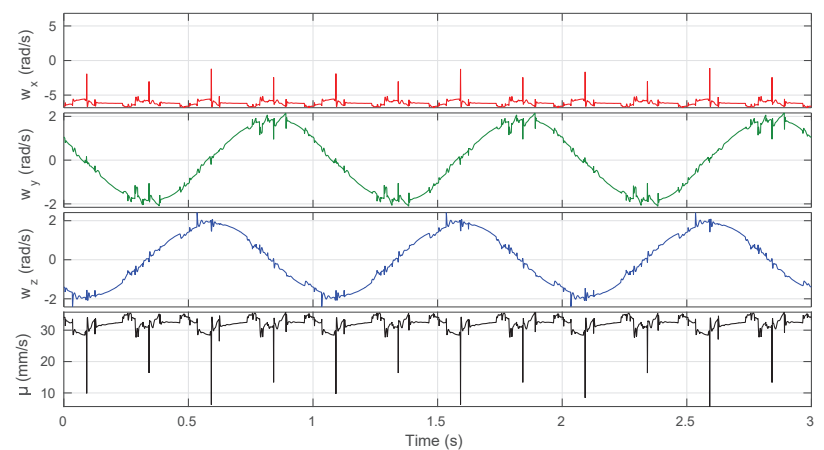

Fig. 9. $\operatorname{Roll}\left(w_{z}\right), \operatorname{pitch}\left(w_{x}\right)$ and $\operatorname{yaw}\left(w_{y}\right)$ rotational speeds of camera and corresponding $\mu$ when straight pointing camera is placed on the forth module of a rolling snake.

rolling gait, it is closer to the legged locomotion characteristics in terms of having rotational oscillations around zero.

1) Head camera looking straight: The motion of camera in swing phase highly fluctuates. Although the maximum motion blur levels are close to the rolling gait, the fluctuating levels of oscillations can be exploited as explained in [16]. For instance camera can be triggered to capture images only when $\mu$ is sufficiently small to obtain only sharper images.

2) Middle camera looking sideways: Changing the camera location to middle and changing the direction changes the motion blur fluctuations significantly. For instance, motion blur remains in lower values for longer durations, even though the maximum values go higher.

3) Middle camera looking up: Placing a camera to look upward on a snake robot can be needed for certain scenarios. For example, when collaboration of ground and aerial robots is needed, upward looking camera can be useful. Moreover, for changing camera from sideways to up does not require any human intervention. Simply exchanging even and odd module amplitudes $\left(A_{e}\right.$ and $\left.A_{o}\right)$ in Eq. (1) is enough to do the side to up transition. Once the camera is pointing upward, mapping between camera coordinates local gyroscope axes would be $\left[\begin{array}{lll}x_{c} & y_{c} & z_{c}\end{array}\right]^{T}=\left[\begin{array}{lll}x_{s 4} & -z_{s 4} & y_{s 4}\end{array}\right]^{T}$. Since, $\mu$ is calculated for the same snake module as in sideways looking camera, only $\mu$ is given in Fig. 12 and it appears to be quite

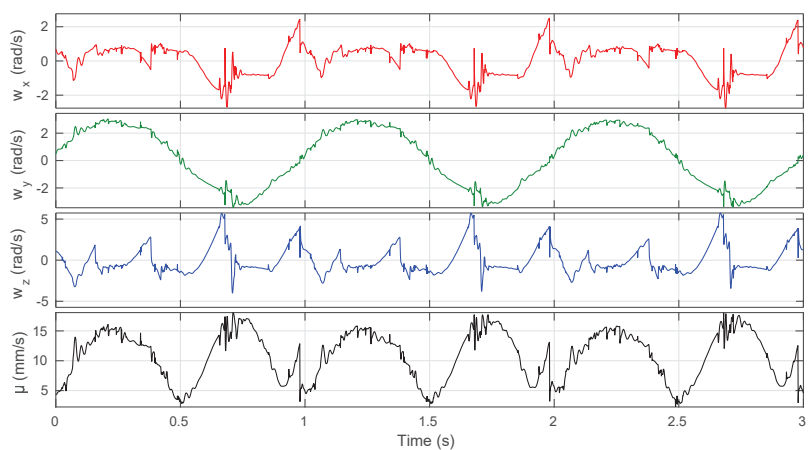

Fig. 10. $\operatorname{Roll}\left(w_{z}\right), \operatorname{pitch}\left(w_{x}\right)$ and yaw $\left(w_{y}\right)$ rotational speeds of camera and corresponding $\mu$ when straight pointing camera is placed on head of a sidewinding snake.

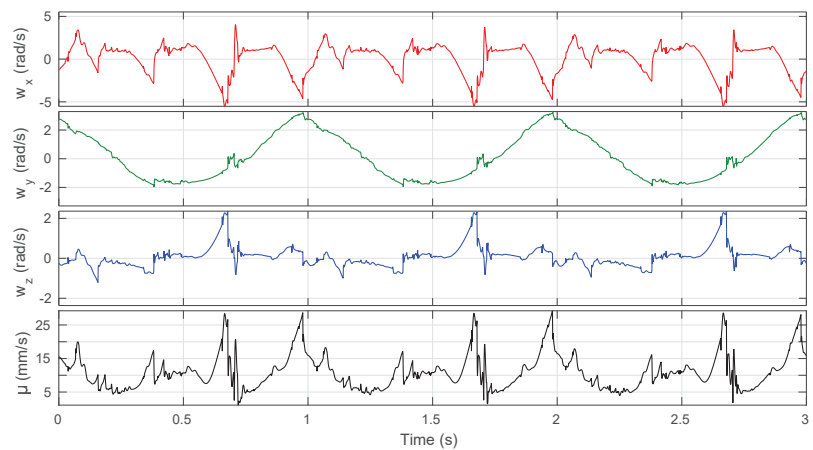

Fig. 11. $\operatorname{Roll}\left(w_{z}\right), \operatorname{pitch}\left(w_{x}\right)$ and yaw $\left(w_{y}\right)$ rotational speeds of camera and corresponding $\mu$ when sideways pointing camera is placed on the forth module of a sidewinding snake.

similar to $\mu$ in Fig. 11

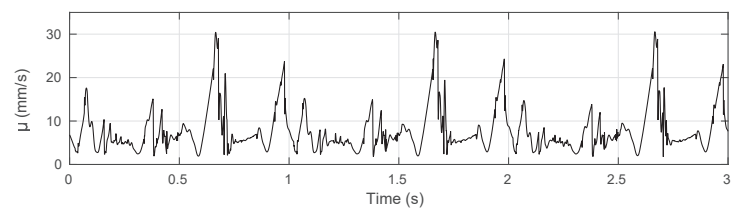

Fig. 12. $\operatorname{Roll}\left(w_{z}\right), \operatorname{pitch}\left(w_{x}\right)$ and yaw $\left(w_{y}\right)$ rotational speeds of camera and corresponding $\mu$ when up pointing camera is placed on the forth module of a sidewinding snake.

\section{Linear progression motion}

Finally the linear progression gives relatively lower motion blur compared to rolling and sidewinding when Fig. 13 and Fig. 14 are examined. The appealing feature of linear progression is that it has significant rotation only in a single axis and small impact related disturbances in the other axes.

1) Head camera looking straight: Rotation of the head corresponds to pitch motion when the camera is looking straight. Hence, the amount of motion blur is still considerable although it is smaller than other gaits. Also the fluctuating behavior allows the acquisition of sharper images at lower values of $\mu$ during locomotion. 


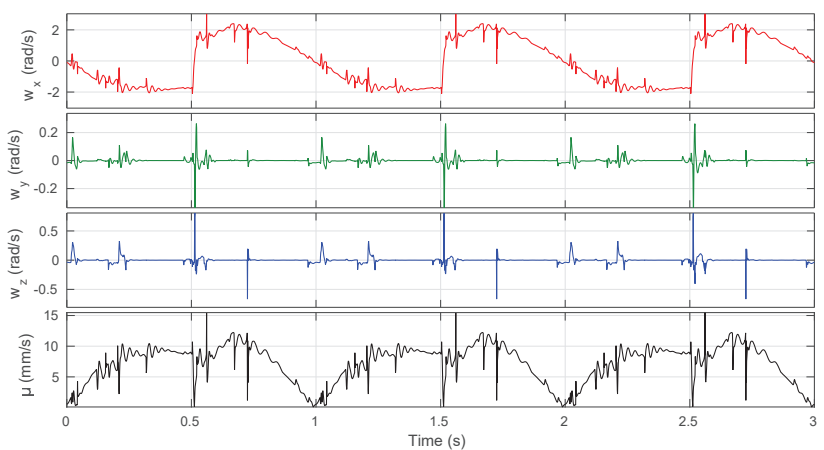

Fig. 13. $\operatorname{Roll}\left(w_{z}\right), \operatorname{pitch}\left(w_{x}\right)$ and yaw $\left(w_{y}\right)$ rotational speeds of camera and corresponding $\mu$ when straight pointing camera is placed on head of a snake doing linear progression.

2) Middle camera looking sideways: The sideways looking camera exhibits the least amount of motion blur among all examined snake gaits since the main rotation is small and it corresponds to roll of camera.

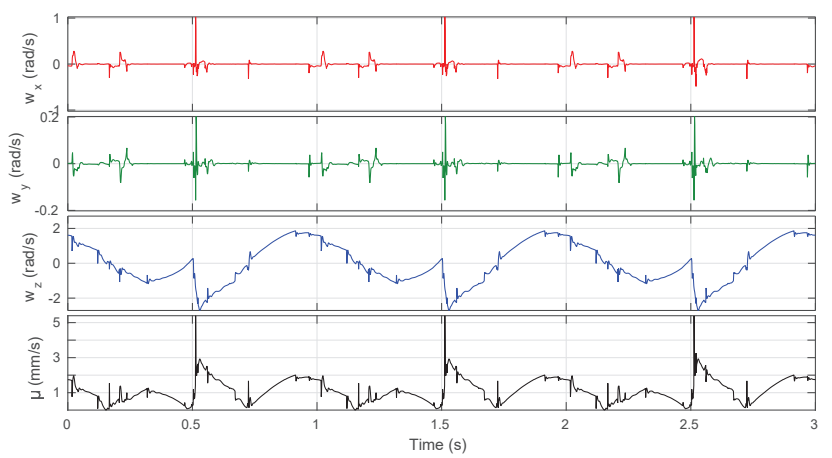

Fig. 14. $\operatorname{Roll}\left(w_{z}\right), \operatorname{pitch}\left(w_{x}\right)$ and yaw $\left(w_{y}\right)$ rotational speeds of camera and corresponding $\mu$ when sideways pointing camera is placed on the forth module of a snake doing linear progression.

3) Middle camera looking up: When the camera is oriented upwards, camera axes changes to $\left[\begin{array}{lll}x_{c} & y_{c} & z_{c}\end{array}\right]^{T}=$ $\left[\begin{array}{lll}-y_{s 4} & x_{s 4} & z_{s 4}\end{array}\right]^{T}$. Snake can also switch camera direction from side to up without external intervention for linear progression. $\mu$ automatically increases since the single rotating axis now corresponds to pitch motion of camera.

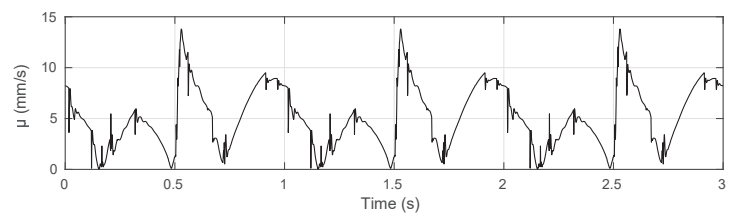

Fig. 15. $\operatorname{Roll}\left(w_{z}\right), \operatorname{pitch}\left(w_{x}\right)$ and yaw $\left(w_{y}\right)$ rotational speeds of camera and corresponding $\mu$ when up pointing camera is placed on the forth module of a snake doing linear progression.

\section{FURTHER DISCUSSIONS AND DEMONSTRATIONS}

Visual data during the snake locomotion can be highly corrupted due to body oscillations and impacts especially
TABLE II

MMBM STATISTICS FOR DIFFERENT SNAKE GAITS

\begin{tabular}{|c|c|c|c|c|c|}
\hline Locomotion & Camera & Min. & Max. & Avg. & Std. Dev. \\
\hline \hline \multirow{3}{*}{ Rolling } & Head & 1.41 & 16.60 & 11.05 & 1.22 \\
\cline { 2 - 6 } & Side & 5.67 & 35.74 & 32.07 & 2.19 \\
\hline \hline \multirow{3}{*}{ Sidewinding } & Head & 2.26 & 18.07 & 10.87 & 4.07 \\
\cline { 2 - 6 } & Side & 1.10 & 29.24 & 10.99 & 5.14 \\
\cline { 2 - 6 } & Up & 1.75 & 30.56 & 7.78 & 5.12 \\
\hline \hline \multirow{3}{*}{$\begin{array}{c}\text { Linear } \\
\text { Progression }\end{array}$} & Head & 0.098 & 15.47 & 7.18 & 3.04 \\
\cline { 2 - 6 } & Side & 0.0072 & 5.39 & 1.12 & 0.65 \\
\cline { 2 - 6 } & Up & 0.0033 & 13.81 & 5.17 & 3.01 \\
\hline
\end{tabular}

when there is not enough light in the environment and exposure time is high. With $\mu$ we have an estimate of motion blur amount on images captured by camera on a snake robot during locomotion. Lower values of $\mu$ shows sharper images if image is captured at that moment. Table II shows that linear progression is very advantageous in terms of average motion blur. On the other hand, having fluctuations on $\mu$ can be exploited to capture relatively sharper images during locomotion. For instance, even if sidewinding and rolling head cameras have similar average motion blur, more variation of sidewinding can be exploited.

In order to give concrete illustration of the motion blur levels and camera motion of examined gaits, consecutive images captured during rolling, sidewinding and linear progression are provided in Fig. 16, Fig. 17 and Fig. 18 respectively. The sharper parts around the middle of rolling images can be seen in Fig. 16. Fig. 17 shows behavior of fluctuating $\mu$ although the illustrated part is mainly located around smaller $\mu$ region. Images can get sharper or more blurry during locomotion. Finally, low to moderate level of blur of linear progression is shown in Fig. 18.

\section{CONCLUSIONS}

We have presented a systematic analysis of camera trajectories during three different types of snake locomotion when the camera is placed on the head pointing straight and in the middle pointing sideways. Camera pose is explicitly computed during different gaits and we were able to analyze implications of different camera locations on field of view coverage during the natural locomotion. We also relate snake motion to motion blur on captured images.

Although linear progression gives sharper images on average, there is no globally optimal location for camera location for all tasks. But, knowing the implications of different camera locations during different gaits, information input in visual tasks can be maximized. For example, if the camera information will be used to steer the robot during sidewinding or rolling, placing the camera on the side is more useful than placing it on the head since head camera will not be pointing to the direction of locomotion. If having multiple onboard cameras is a possibility, we can place them as to obtain maximal benefits. The proposed analysis can be used for designing new robots for a given desired gait or 


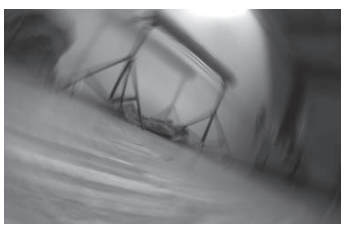

(a)

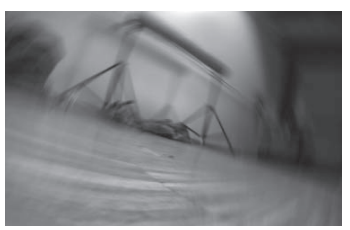

(b)

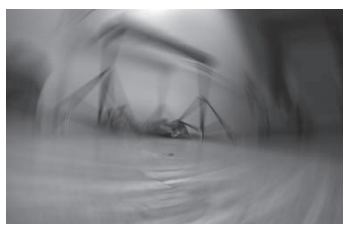

(c)

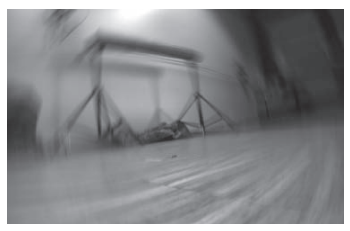

(d)

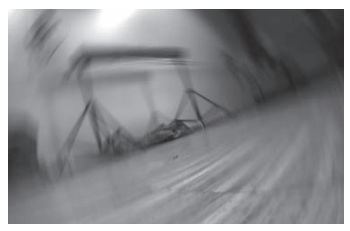

(e)

Fig. 16. Consecutive images captured during rolling gait by camera mounted on head of the robot pointing straight.

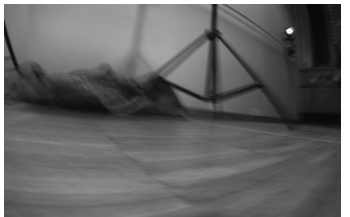

(a)

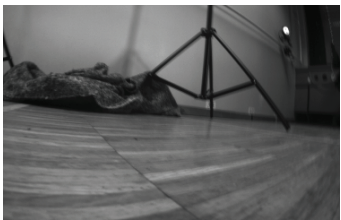

(b)

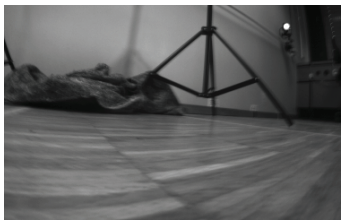

(c)

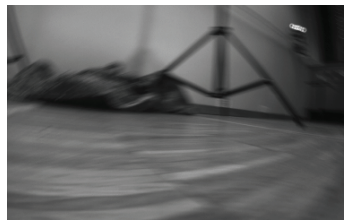

(d)

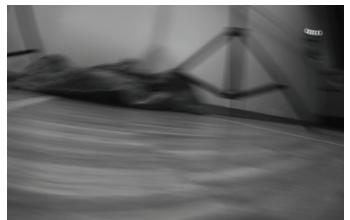

(e)

Fig. 17. Consecutive images captured during sidewinding gait by camera mounted on head of the robot pointing straight.

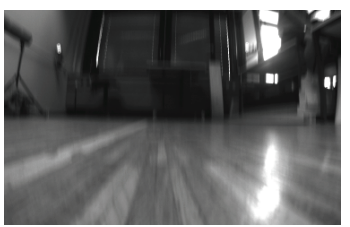

(a)

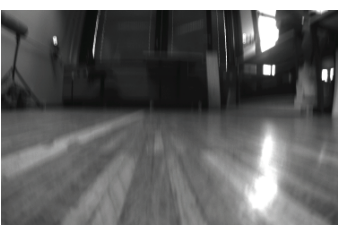

(b)

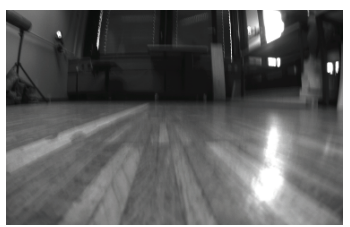

(c)

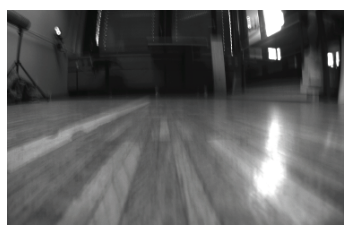

(d)

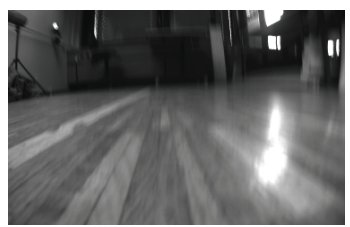

(e)

Fig. 18. Consecutive images captured during linear progression gait by camera mounted on head of the robot pointing straight.

to choose suitable gaits for already existing robots that are equipped with cameras. Ultimately, it will contribute to the quality of visual SLAM during snake locomotion.

\section{ACKNOWLEDGMENT}

This work was supported by the Swiss National Science Foundation through the National Centre of Competence in Research (NCCR) Robotics and Fundação para a Ciência e Tecnologia (FCT) agency of Ministry for Education and Science of Portugal under the FCT doctoral grant $[\mathrm{PD} / \mathrm{BD} / 105781 / 2014]$ and $\mathrm{FCT}$ project grant [UID/EEA/50009/2013].

\section{REFERENCES}

[1] R. R. Murphy, Disaster Robotics. The MIT Press, 2014.

[2] J. Huff, R. Voyles, and S. Tadokoro, "Explosion proof active scope camera," in Safety, Security, and Rescue Robotics (SSRR), 2012 IEEE International Symposium on, Nov 2012, pp. 1-3.

[3] K. Melo, J. Leon, A. di Zeo, V. Rueda, D. Roa, M. Parraga, D. Gonzalez, and L. Paez, "The Modular Snake Robot Open Project: Turning animal functions into engineering tools," in Safety, Security, and Rescue Robotics (SSRR), 2013 IEEE International Symposium on, Oct 2013, pp. 1-6.

[4] S. H. Hiroya Yamada, Shunichi Takaoka, "A snake-like robot for realworld inspection applications (the design and control of a practical active cord mechanism)," Advanced Robotics, vol. 27, no. 1, pp. 4760, 2013.

[5] C. Wright, A. Buchan, B. Brown, J. Geist, M. Schwerin, D. Rollinson, M. Tesch, and H. Choset, "Design and architecture of the unified modular snake robot," in Robotics and Automation (ICRA), 2012 IEEE International Conference on, May 2012, pp. 4347-4354.
[6] P. Liljeback, O. Stavdahl, K. Pettersen, and J. Gravdahl, "Mamba a waterproof snake robot with tactile sensing," in Intelligent Robots and Systems (IROS 2014), 2014 IEEE/RSJ International Conference on, Sept 2014, pp. 294-301.

[7] D. Rollinson, Y. Bilgen, B. Brown, F. Enner, S. Ford, C. Layton, J. Rembisz, M. Schwerin, A. Willig, P. Velagapudi, and H. Choset, "Design and architecture of a series elastic snake robot," in Intelligent Robots and Systems (IROS 2014), 2014 IEEE/RSJ International Conference on, Sept 2014, pp. 4630-4636.

[8] X. Wu and S. Ma, "Sensor-driven neural controller for self-adaptive collision-free behavior of a snake-like robot," in Robotics and Automation (ICRA), 2011 IEEE International Conference on, may 2011, pp. 191-196.

[9] M. Tesch, J. Schneider, and H. Choset, "Expensive multiobjective optimization for robotics," in Robotics and Automation (ICRA), 2013 IEEE International Conference on, May 2013, pp. 973-980.

[10] J. Florez, F. Calderon, and C. Parra, "Video stabilization taken with a snake robot," in Image, Signal Processing, and Artificial Vision (STSIVA), 2013 XVIII Symposium of, Sept 2013, pp. 1-5.

[11] K. Melo and L. Paez, "Experimental determination of control parameter intervals for repeatable gaits in modular snake robots," in Safety, Security, and Rescue Robotics (SSRR), 2014 IEEE International Symposium on, Oct 2014, pp. 1-7.

[12] W. Zhen, C. Gong, and H. Choset, "Modeling rolling gaits of a snake robot," in Robotics and Automation (ICRA), 2015 IEEE International Conference on. IEEE, 2015, pp. 3741-3746.

[13] S. Cho and S. Lee, "Fast motion deblurring," ACM Trans. Graph., vol. 28 , no. 5 , pp. $145: 1-145: 8$, Dec. 2009.

[14] J. Hilkert, "Inertially stabilized platform technology concepts and principles," Control Systems, IEEE, vol. 28, no. 1, pp. 26-46, Feb 2008

[15] P. Liljebäck, K. Y. Pettersen, Ø. Stavdahl, and J. T. Gravdahl, "Future research challenges of snake robot locomotion," in Snake Robots. Springer, 2013, pp. 287-291.

[16] M. Mutlu, A. Saranli, and U. Saranli, "A real-time inertial motion blur metric: Application to frame triggering based motion blur minimization," in Robotics and Automation (ICRA), 2014 IEEE International Conference on, May 2014, pp. 671-676. 